\title{
Providing Variable TXOP for IEEE 802.11e HCCA Real-Time Networks
}

\author{
Gabriele Cecchetti*, Anna Lina Ruscelli ${ }^{\dagger}$, Antonia Mastropaolo ${ }^{\ddagger}$, and Giuseppe Lipari ${ }^{\S}$ \\ ReTis Lab - TeCIP \\ Scuola Superiore Sant'Anna \\ Pisa, Ialy \\ Email: *g.cecchetti@sssup.it, †a.ruscelli@sssup.it, ${ }^{\ddagger}$ antoniamastropaolo@gmail.com, §g.lipari@sssup.it
}

\begin{abstract}
Quality of Service (QoS) provided by the IEEE 802.11e amendment and by the proposed HCF Controlled Channel Access (HCCA) reference scheduler is tailored for Constant Bit Rate traffic streams. Moreover the numerous alternative scheduling algorithms are not suitable to serve Variable Bit Rate (VBR) traffic streams with the required QoS and real-time guarantees.

This paper presents Immediate Dynamic TXOP HCCA (IDTH), a new scheduling algorithm based on a bandwidth reclaiming mechanism suitable to cooperate with a HCCA real-time scheduler. IDTH recovers the portion of the transmission time unused by the scheduled stations to provide a further capacity for the next variable bit rate traffic streams. The transmission opportunity of the next scheduled station is assigned considering the available spare resources and the previously used ones. The scheduling analysis and the simulations results show that IDTH is suitable to reduce the delay experienced by VBR traffic streams, to efficiently deal with the variability of multimedia traffic and to avoid waste of resources.
\end{abstract}

Index Terms-Quality of Service, real-time scheduling algorithms, Wireless LAN.

\section{INTRODUCTION AND RELATED WORKS}

The IEEE 802.11e amendment [16] to the IEEE 802.11 reference standard [15] for Wireless Local Area Networks (WLAN) has been proposed to improve the Quality of Service (QoS) support as required by multimedia applications like Voice over IP (VoIP), multimedia streaming, High Definition $\mathrm{TV}$, online gaming. It introduces a traffic differentiation by means of two additional Medium Access Control (MAC) functions. Hybrid coordination function Controlled Channel Access (HCCA) introduces a polling mechanism with a parameterized QoS, whereas Enhanced Distributed Channel Access (EDCA) function is based on a contention medium access with a prioritized QoS. The IEEE 802.11e HCCA proposed reference scheduler draws some guidelines for the computation of the main protocol parameters: the polling period, named Service Interval (SI), and the transmission duration Transmission Opportunity (TXOP). Both of them are computed as fixed values during the admission control phase and are recomputed only if a new Traffic Stream (TS) is admitted to transmit. The reference scheduler suggests that SI, that is the same for all admitted TSs, has to be less than the minimum Maximum Service Interval (MSI), thus the constraints about the period of all traffic streams are respected, and less than the beacon interval T, thus each QoS Station (QSTA) is polled at least once during the beacon interval. TXOP, globally assigned to a QSTA, is computed as the maximum time to transmit at the minimum physical rate the total amount of bits that can arrive during $S I$. The admission control condition that has to be respected when admitting a new traffic stream is:

$$
\frac{T X O P_{k+1}}{S I}+\sum_{i=0}^{k} \frac{T X O P_{i}}{S I} \leq \frac{T-T_{C P}}{T} \leq 1 .
$$

where $k$ is the number of admitted streams, $k+1$ is the index of the newly admitted stream, and $T_{C P}$ is the EDCA duration.

However, since $S I$ and $T X O P$ are computed considering worst case conditions, the admission control is too severe and the resource management is not optimal. Furthermore numerous theoretical and simulative studies about the HCCA reference scheduler [8], [13], [23], [28] shown that it is suitable to serve only Constant Bit Rate (CBR) traffic, whereas it is unable to efficiently manage Variable Bit Rate (VBR) TSs, since all different TSs are polled with the same period and are served with the same computation time. Hence many scheduling algorithms alternative to the reference one have been proposed to improve the HCCA QoS provisioning [10], [11], [22], [28], but the support of real-time guarantees due to temporal requirements has been investigated only by few works [4].

Fair HCF (FHCF) [1] is an example of scheduler using a queue length model to compute variable TXOPs. Instead, Feedback Based Dynamic Scheduler (FBDS) [3] uses a closed loop feedback control to limit the maximum delay. Some works have investigated the use of the IEEE 802.11e HCCAEDCA Mixed Mode (HEMM), that allows the use of both HCCA and EDCA resources, to improve the HCCA QoS support. Some of them use a channel model [18], or optimization techniques to find the optimal HCCA-EDCA duration ratio [26], or a dynamic tune of HCCA and the EDCA durations for adapting to the different type of traffic [19]. The Overboost local node scheduler [25], that can collaborate with any type of centralized HCCA scheduling algorithm, moves the TSs traffic exceeding the assigned TXOP from the HCCA queue to the higher priority Access Category EDCA queue. Some algorithms are based on the use of deadlines to model temporal requirements. Scheduling Estimated Transmission Time - Earliest Due Date (SETT-EDD) [12] algorithm uses a token 
bucket scheme to vary TXOPs over time according to the station requirements. In [9] a timer-based scheduler computes the transmissions deadlines as the smallest between the downlink and uplink ones, and Earliest Deadline First (EDF) [21] algorithm schedules traffic streams. Real-Time HCCA (RTH) scheduler [6] manages $T X O P$ as critical section by using Stack Resource Policy (SRP) algorithm [2] and EDF to schedule TS transmissions. Adaptive Resource Reservation Over WLANs (ARROW) [27] dynamically computes each $T X O P$ by taking into account the actual buffered TSs data at the beginning of the polling. Its derivation ApplicationAware Adaptive HCCA Scheduler [17] distinguishes uplink and downlink schedulers and the former assigns each QSTA a minimum and a maximum $S I$, adapted to application and network conditions and to the buffered traffic. Wireless Capacity Based Scheduler (WCBS) [5] dynamically updates the EDFbased polling list and uses static and dynamic parameters to adapt the transmissions scheduling to the TSs characteristics. During the admission control the budget $Q_{i} \equiv T X O P_{i}$, and the period $P_{i} \equiv S I_{i}$, are statically assigned to each $\mathrm{TS}_{i}$, taking into account its Traffic Specification $\left(\mathrm{TSPEC}_{i}\right)$. The admission control test is defined as:

$$
\frac{T X O P_{k+1}}{S I_{k+1}}+\sum_{i=0}^{k} \frac{T X O P_{i}}{S I_{i}} \leq \frac{T-T_{C P}}{T} \leq 1 .
$$

Instead, the dynamic parameters are used during the scheduling of each $\mathrm{TS}_{i}$ : the remaining time $c_{i}$ from previous transmission assigned to $\mathrm{TS}_{i}$ for its next transmission, the absolute deadline $d_{i}$ before $Q_{i}$ has to be exhausted, the next polling time $p_{i}$, and the traffic stream state (transmitting, active, polling, idle). Some algorithms have introduced a bandwidth reclaiming to recover unused resources and to reduce the delay in the case of VBR traffic [20], [24], [7].

Anyway, despite the numerous HCCA improved schedulers, QoS provisioning for VBR traffic with real-time requirements is an open issue. In particular, the lack of flexibility in the resource assignment, not suitable to follow the traffic variability, produces a waste of resources. Indeed, when data rate drops down, the transmission duration of a station can be shorter that the assigned one. Thus, when the station frees the medium, the control is recovered by QoS Access Point (QAP) that polls the next station in the polling list and, if no bandwidth reclaiming schemes are used, the unspent transmission time is lost. Instead, when data rate goes up, the delay increases since the transmission opportunity is not sufficient to dispatch enqueued packets. Hence, why do not use these spare resources to provide a further portion of the transmission time to send VBR traffic bursts and to absorb traffic variability?

This is the basic idea of Immediate Dynamic TXOP HCCA (IDTH), a novel scheduler presented in this paper. It integrates a mechanism for bandwidth reclaiming and for estimation of the required resources into a HCCA real-time scheduler. IDTH assigns the current transmission time considering two variables: $a$ ) the portion of TXOP unused by polled stations, and b) the effective transmission time used by the station during its previous polling. IDTH computes a dynamic current TXOP, without interfere with the admission control nor with the policy of the real-time HCCA scheduler. We will show through analytical study and simulation that the proposed scheduler is suitable to follow the traffic variability, to efficiently manage bursts of traffic, to reduce the delay experienced by VBR traffic streams and to avoid waste of resources. The rest of the paper is organized as follows: in Section II the proposed scheduler is described. In Section III some properties of the scheduler are analytically evaluated, whereas Section IV shows its performance through simulations. Finally, in Section V we draw some conclusions.

\section{IMMEDIATE DYNAMIC TXOP HCCA}

Variable bit rate traffic stresses the scheduling behavior of the numerous HCCA algorithms since it requires a flexible resource management. For instance, EDF-based algorithms are suitable to efficiently manage only CBR traffic, whereas they are under-performing in the case of VBR TSs [4].

Indeed most schedulers compute TXOP during the admission control phase considering mean values of traffic parameters and QoS requirements. Thus, in the case of data rate higher than the mean data rate, the scheduler is not suitable to efficiently manage real-time TSs, increasing their delay, whereas, in the case of lower data rate, the accorded capacity is not exhausted and it is lost. For instance, in WCBS, see Section I, the unused portion of TXOP is maintained for the next polling of the same QSTA but, if it is less than the minimum time needed to send a Service Data Unit (SDU), it is lost.

Immediate Dynamic TXOP HCCA (IDTH) is based on a reclaiming scheme to overcome the lack of scheduling flexibility in the case of VBR TSs. It integrates a reclaiming algorithm in a real-time HCCA scheduler, like WCBS. In particular, it takes action at the polling time of a station when the current TXOP is assigned, without modifying the admission control, and its computation of TXOP and SI and without impacting on the scheduling policy, as we will show in Section III and in Section IV-A.

IDTH keeps the portion of unused allocated resources and adds that to the currently assigned transmission time of the next scheduled station in order to better follow the traffic variability and to absorb the bursts of traffic. Moreover, it combines this reclaiming scheme with a further policy to take into account the effective used resources. This avoids to assign too much resources to stations that have shown, in the previous polling, a low date rate and that do not need, at the present time, an additional capacity.

Thus, when a station QSTA $_{i}$ ends its transmission, IDTH stores $t_{\text {effi }}$, its effectively used transmission time, that will be considered at its next polling. Then, whenever the QAP polls the next station extracted from the EDF-ordered polling list, IDTH computes $T_{\text {spare }}$, the spare time from the previous transmissions, as follows:

$$
T_{\text {spare }}=t_{\text {end }}-t_{\text {stop }},
$$


where $t_{\text {end }}=t_{p}+T X O P$ is the ending transmission time when TXOP is completely exhausted ( $t_{p}$ is the polling time), and $t_{\text {stop }}$ is the actual finishing transmission time.

The current assigned $T X O P_{i}^{\prime}$ is computed as:

$$
\text { TXOP }_{i}^{\prime}= \begin{cases}T X O P_{i_{A C}} & \text { if } T_{\text {spare }}=0 \\ t_{\text {effi }}+T_{\text {spare }} & \text { if } T_{\text {spare }}>0\end{cases}
$$

where $T X O P_{i_{A C}}$ is the transmission opportunity of $\mathrm{QSTA}_{i}$ computed during the admission control phase. Figure 1 shows some examples of IDTH scheduling, distinguishing the case of $T_{\text {spare }} \equiv 0(a)$ and $T_{\text {spare }} \neq 0(b)$ at the end of a Contention Free Period (CFP). In particular, in Eq. 2 setting $T X O P_{i}^{\prime}=$ $T X O P_{i_{A C}}$ when $T_{\text {spare }}=0$ is because assigning $t_{\text {effi }}$, as in the case of $T_{\text {spare }} \neq 0$, can produce a not correct behavior; we will motivate this consideration in Section III.

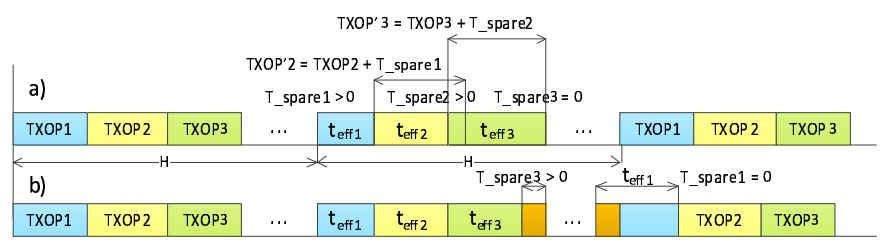

Fig. 1. IDTH scheduling examples.

\section{SCHEDULER ANALYSIS}

In this section an analytical study of IDTH is described with the aim to investigate if and how the real-time properties of the HCCA scheduler are changed when integrating IDTH. First of all it is shown how its settings allow to follow traffic variations. Then it is analyzed how the IDTH mechanism impacts on the admission control feasibility condition of the HCCA scheduler. Finally it is investigated if the respect of the real-time requirements of admitted stations is jeopardized by IDTH.

Proposition 1: Setting $T X O P_{i}^{\prime}=T X O P_{i_{A C}}$ when $T_{\text {spare }}=0$ allows to better follow data rate changes of VBR traffic streams.

Proof: Without loss of generality Fig. 2 shows a scheduling example of a QSTA with VBR TSs, where both situations of decreasing and increasing data rate are analyzed if we set $\mathrm{TXOP}_{i}^{\prime}=t_{\text {effi }}$. When data rate decreases, see Fig. 2a), if we suppose that at $i^{\text {th }}$ polling the station does not use the whole assigned $T X O P_{i}$, thus $t_{\text {eff } i} \neq T X O P_{i}$. At $(i+1)^{t h}$ polling IDTH computes the current $T X O P_{i+1}^{\prime}=t_{\text {ef } f i}$, (for reasons of simplicity we assume that $T_{\text {spare }}$ from previous stations is equal to zero, but general results about the working of IDTH do not change if we relax this assumption). If once again the transmission time is not completely exhausted, thus $0<t_{\text {eff }(i+1)}<t_{\text {eff } i}$. If the data rate continues to decrease, this behavior persists and allows to save resources. But if the data rate variation changes and begins increasing, as it is common for VBR TSs, at the next transmission opportunity the station can exhaust the whole assigned $T X O P_{i+2}^{\prime} \equiv t_{\text {eff }(i+1)}$ and the actual used transmission time, assigned as next transmission time, is $t_{\text {eff }(i+2)}=t_{\text {eff(i+1) }}$. No more resources cannot be assigned and this version of IDTH generates an irreversible decreasing of $t_{e f f}$, whose trend cannot be inverted. Thus it is not possible to follow traffic variations and discarded packets and delay increase. Instead, as expected, when the date rate increases, see Fig. 2b), the maximum transmission time is assigned and, if the data rate variation changes its sign, the mechanism allows to reduce the allocated capacity. Hence assigning $T X O P_{i_{A C}}$ when $T_{\text {spare }}=0$ introduces a more performing tracking mechanism tailored to VBR traffic streams. The use of $T_{\text {spare }}$ can partially recover this misbehavior, providing a further slot time that can increase the transmission opportunity, but its randomness does not allow a predictable scheduling behavior.

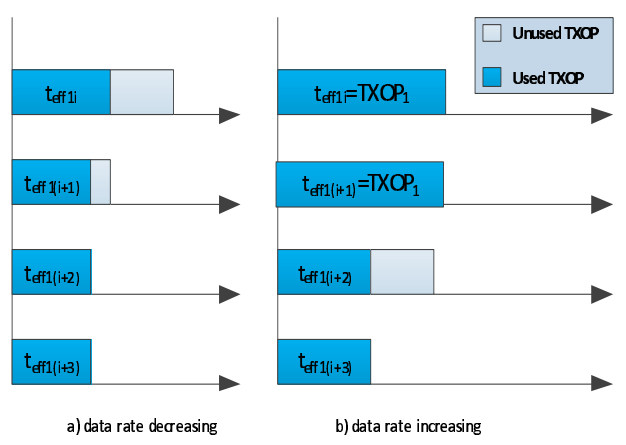

Fig. 2. IDTH scheduling example if setting $T X O P_{i}^{\prime}=t_{\text {eff } i}$.

Theorem 1: The admission control feasibility test remains valid when the IDTH scheduler is used.

Proof: For reasons of simplicity in the admission control feasibility test expressed by Eq. 1, we assume to consider only the portion of time assigned to HCCA:

$$
\sum_{i=0}^{k} T X O P_{i} \leq T-T_{C P}
$$

We study the general situation illustrated in Fig. 1 where three stations are scheduled and a chain of $T_{\text {spare }}$ assignment is spread between that, and we distinguish a set of different cases in order to provide a global evaluation of the IDTH scheduling. Moreover, it is important to highlight that the bandwidth reclaiming scheme implies an instantaneous check of the admission control condition during each Controlled Access Phase (CAP), where the assigned TXOP' of each QSTA is recomputed considering the behavior of the preceding stations and not only adopting the value from the admission control.

Case $a$ The propagation of $T_{\text {spare }}$ ends with $\mathrm{QSTA}_{3}$ that exhausts all spare resources and the admission control updated step-by-step during the scheduling is as follows:

$$
\begin{aligned}
& t_{\text {eff } 1}+T_{2}^{\prime}+T_{3}^{\prime}=T_{1}-\tau_{1}+T_{2}+\tau_{1}-\tau_{2}+T_{3}+\tau_{2}= \\
& T_{1}+T_{2}+T_{3} .
\end{aligned}
$$

where $T_{i}=T X O P_{1}$ and $T_{i}^{\prime}=T X O P_{i}$ is the current assigned TXOP of $\mathrm{QSTA}_{i}$ and $\tau_{i}$ is $T_{\text {sparei }}$ derived from $\mathrm{QSTA}_{i}$. 
Case $b$ The general situation of propagation between CFP is illustrated: $\mathrm{QSTA}_{1}$ receives $T_{\text {spare } 3}=\tau_{3}$ from the previous CAP and it does not exhausts its transmission time $T X O P_{1}$, and the same happens for $\mathrm{QSTA}_{2}$ and $\mathrm{QSTA}_{3}$. Thus it is necessary to evaluate the admission control condition $A C$ during the two consequent $i$ and $i+1$ CAP:

$$
\begin{aligned}
A C_{i} & =T_{1}-\tau_{1}+T_{2}+\tau_{1}-\tau_{2}+T_{3}+\tau_{2}-\tau_{3} \\
& =T_{1}+T_{2}+T_{3}-\tau_{3} \\
& \leq T_{1}+T_{2}+T_{3} . \\
A C_{i+1}= & t_{e f f 1}+\tau_{1}+\tau_{3}+T_{2}+\tau_{1}-\tau_{2}+T_{3}+\tau_{2}-\tau_{3}^{\prime} \\
= & T_{1}+\tau_{3}-\tau_{1}+T_{2}+\tau_{1}-\tau_{2}+T_{3}+\tau_{2}-\tau_{3}^{\prime} \\
\leq & T_{1}+T_{2}+T_{3}+\tau_{3}-\tau_{3}^{\prime} .
\end{aligned}
$$

In this situation there is only an advance of $(i+1)^{t h}$ equal to $\tau_{3}$ and the assignment of already allocated but unused resources, without violating the global admission control test.

Shown that IDTH does not impact on the admission control, it is interesting to analyze if the increment of TXOP by adding $T_{\text {spare }}$ can jeopardize the respect of deadlines.

Theorem 2: The IDTH mechanism allows to continue meeting deadlines.

Proof: First of all it is important to highlight that in wireless networks the MAC protocol can introduce some further constraints that integrate the real-time scheduling. In particular, HCCA manages the medium access of stations by the use of fixed interframe space intervals (Short Interframe Space (SIFS) and PCF Interframe Space (PIFS)); this rule avoid the presence of idle blocking time between polled stations transmissions, as in general it could happen in real-time systems, where the addition of a further slot of transmission time could raise a deadline miss.

Since IDTH stores $T_{\text {spare }}$ between different CAP of a CFP or between different CFP, we analyze two different situations when, for instance, three stations are polled during a CAP and: a) there is not $T_{\text {spare }}$ propagation between two contiguous CAP, since the last polled station exhausts its TXOP, see Fig. 1a), and b) there is $T_{\text {spare }}$ propagation between two contiguous CAP, see Fig. 1b).

Case $a$ When the last polled station during a CAP does not live spare resources, $T_{\text {spare }}=0$ and IDTH simply assigns $T X O P=T X O P_{A C}$ of the first polled QSTA. Moreover, during the CAP the distribution of $T_{\text {spare }} \neq 0$ simply moves some resources, yet allocated, from a station to the follower thus there is only an advance of the polling time and a re-distribution of resources computed during the admission control, without the risk to extend the transmission beyond the delay bound, causing a deadline miss. Instead, if always during a CAP $T_{\text {spare }}=0$ the original values of TXOP calculated during the admission control are assigned and, obviously, there is no deadline miss.

Case $a$ When $T_{\text {spare }} \neq 0$ between two contiguous CAP, i.e. the last polled station does not exhaust its $T X O P$, this causes an early polling of the first QSTA during the new CAP of the same time interval $T_{\text {spare }}$. Thus also in this case there is not deadline miss. The case of propagation between different CFP is analogue to the illustrated ones, with the difference that now there is an advance of Contention Period (CP) and CFP.

\section{PERFormance ANAlysis}

In this section the performance of the proposed IDTH algorithm are analyzed versus WCBS and reference scheduler with the aim to evaluate the impact of IDTH on the scheduling in terms of efficient resource management, experienced delay and discarded packets due to delay bound expiration.

We assume ideal conditions where QSTAs communicate directly without hidden node problem and RTS/CTS mechanism, MAC level fragmentation and multirate support are disabled. The Physical layer is specified in the IEEE 802.11g standard where OFDM (Orthogonal Frequency-Division Multiplexing) is the mandatory modulation scheme, see Table I. Performance

TABLE I

MAC/PHY SIMULATION PARAMETERS.

\begin{tabular}{lrlr}
\hline Parameters & Value & Parameters & Value \\
\hline SIFS $(\mu s)$ & 10 & PLCP header $(b)$ & 24 \\
DIFS $(\mu s)$ & 28 & Preamble $(b)$ & 72 \\
PIFS $(\mu s)$ & 19 & Data Rate $(M b i t / s)$ & 54 \\
Slot Time $(\mu s)$ & 9 & Basic Rate $(M b i t / s)$ & 1 \\
\hline
\end{tabular}

are evaluated through $n s-2$ network simulator by running independent replications of $700 \mathrm{~s}$ with a warm-up time of $100 \mathrm{~s}$ until the $95 \%$ confidence interval is reached for each measure. Network scenario is composed by one station with G.729A VoIP traffic whose parameters are shown in Table II, five stations transmitting video streaming applications and one a videoconference. Video streaming traffic is composed by

TABLE II

G.729A VOIP PARAMETERS

\begin{tabular}{lrlr}
\hline Parameter & Value & Parameter & Value \\
\hline Frame size $(B)$ & 10 & Payload size $(B)$ & 20 \\
Frames per packet & 2 & IP/UDP/RTP & \\
Period $(s)$ & 0.02 & Header size $(B)$ & 40 \\
Data rate $(\mathrm{kb} / \mathrm{s})$ & 24 & SDU size $(B)$ & 60 \\
\hline
\end{tabular}

high quality MPEG4 trace files of 60 minutes (Jurassic Park (VS1), Silence of the lambs (VS2), Mr. Bean (VS3), Die hard III (VS4), Robin Hood (VS5)) [14]. The videoconference is simulated with the pre-encoded trace file LectureHQ-Reisslein. The corresponding parameters are listed in Table III. Moreover one data station operates in asymptotic condition, (i.e. it has always traffic to transmit) and sends SDU of 1500 bytes through Distributed Coordination Function (DCF).

\section{A. Efficiency analysis}

The efficient resource management is evaluated considering the null rate, i.e. the sending rate of $\mathrm{CF}-\mathrm{Null}$ packets as answer to a polling when the station has no data to transmit, since it is due to an uncorrect polling time choice and can increase the network overhead. Fig. 3 shows as IDTH and WCBS can reduce the null rate with respect to the reference scheduler until a zero null rate since they assign $S I$ and 
TABLE III

VIDEO STREAMING AND VIDEO VONFERENCE PARAMETERS

\begin{tabular}{lrrr}
\hline Parameter & VS1 & VS2 & VS3 \\
\hline Mean frame size (B) & 3800 & 2900 & 2900 \\
Maximum frame size (B) & 11386 & 22239 & 15251 \\
Period (s) & 0.040 & 0.040 & 0.040 \\
Mean data rate (b/s) & 770000 & 580000 & 580000 \\
Maximum data rate (b/s) & 3300000 & 4400000 & 3100000 \\
\hline Parameter & VS4 & VS5 & VC \\
\hline Mean frame size (B) & 3500 & 4600 & 3800 \\
Maximum frame size (B) & 16960 & 16550 & 11386 \\
Period (s) & 0.040 & 0.040 & 0.040 \\
Mean data rate (b/s) & 700000 & 910000 & 770000 \\
Maximum data rate (b/s) & 3400000 & 3300000 & 3300000 \\
\hline
\end{tabular}

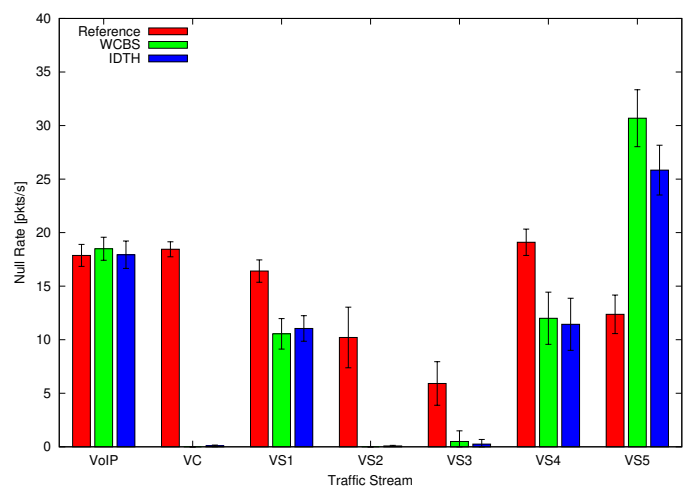

Fig. 3. Null rate

TXOP considering the QSTAs requirements. Fig. 4 confirms that, when the IDTH mechanism is integrated with an HCCA centralized scheduler, it does not modify its scheduling policy nor the computation of protocol parameters like the polling interval. From this point of view its effect can only be an early polling and this justifies similar values of the null rate with respect to WCBS. Moreover Fig. 5, referred to

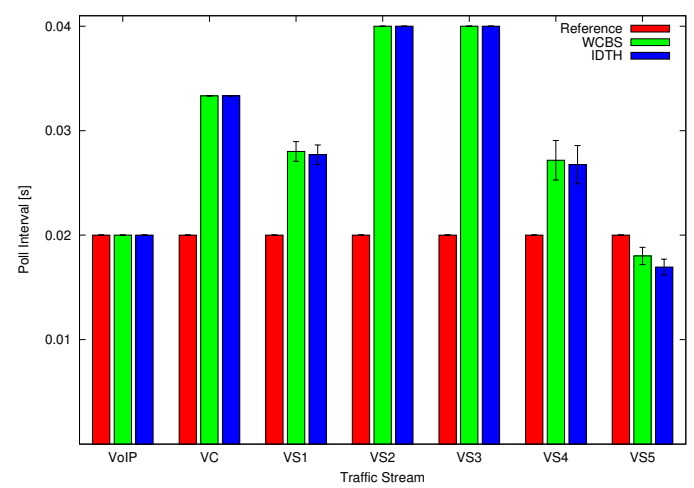

Fig. 4. Polling interval.

the $99^{\text {th }}$ percentile of queue length, illustrates that IDTH can improve the resource assignment and reduce the queue length of waiting packets. This is clear especially looking at highly variable bit rate TSs, for example VS2, that shows a considerable queue reduction when IDTH is used, about $75 \%$ in comparison with the reference scheduler, and about
$50 \%$ in comparison with WCBS. Indeed IDTH allows to vary the current TXOP during the transmission, to decrease wasted resources and to better distribute the bandwidth.

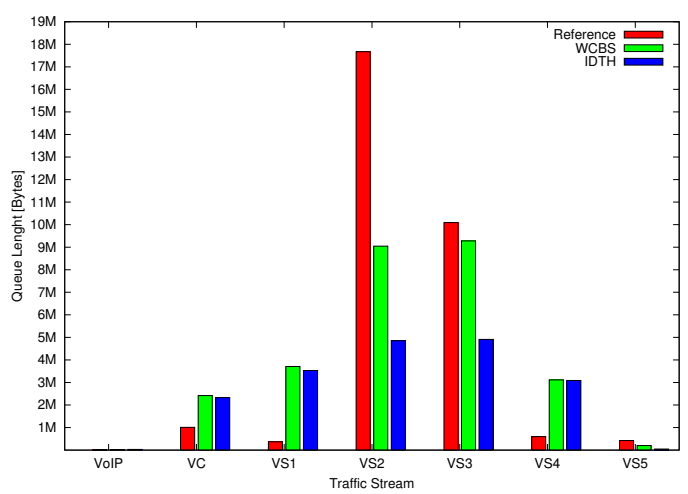

Fig. 5. Queue length.

\section{B. Delay analysis}

The real-time behavior of the schedulers is evaluated considering the access delay, defined as the interval between the time when a packet reaches the MAC level and when the corresponding ACK is received. Fig. 6 shows that, in general, IDTH reduces the mean access delay with respect to WCBS and reference schedulers, and this confirms the analytical results illustrated in Section III. In particular, its effect is more relevant with highly variable bit rate traffic streams, since in this case $T_{\text {spare }}$ has big variations and the assigned current $T X O P^{\prime}$ can be different respect to $T X O P_{A C}$ used by WCBS and reference schedulers. Thus the streams that take more advantage from the use of IDTH are the most variable ones. The Cumulative Distribution Function (CDF) of the access delay experienced by VS3 TS, shown in Fig. 7, confirms these considerations.

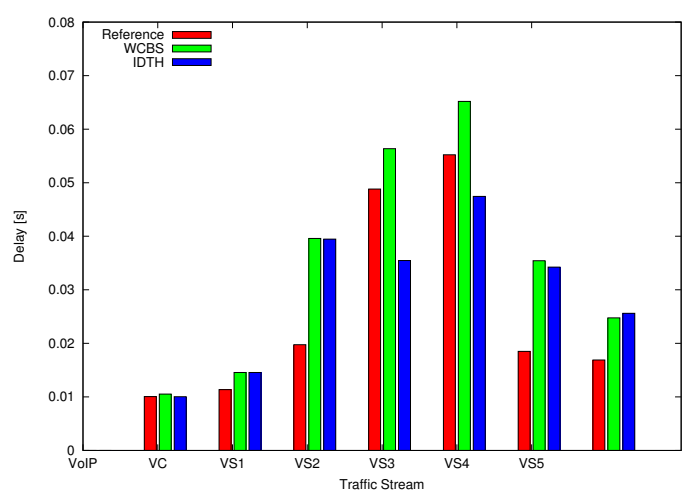

Fig. 6. Mean access delay.

\section{Discarded packets analysis}

In ideal conditions packets are discarded only if their waiting time overcomes their delay bound, thus this analysis highlights the effects of IDTH on the real-time performance. 


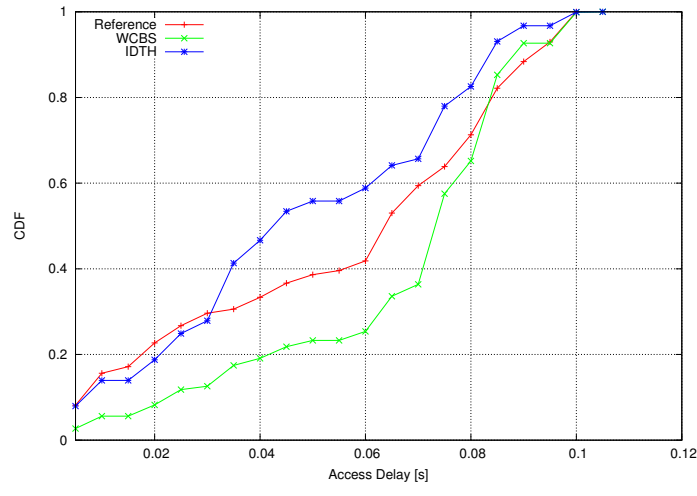

Fig. 7. CDF of access delay of VS3 traffic stream.

The bandwidth reclaiming scheme allows to reduce the time a packet remains into the queue, as shown by queues length and delay analysis, see Section IV-A. According to this the delay is reduced and, in the same way, discarded packets are reduced, as shown in Fig. 8.

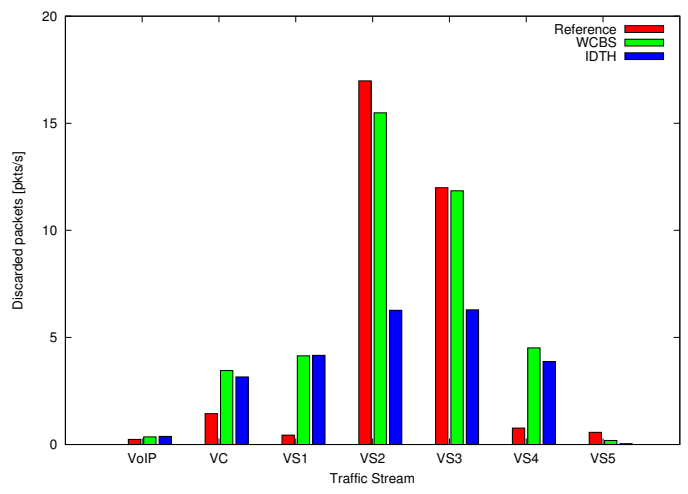

Fig. 8. Discarded packets

\section{CONCLUSIONS}

In this paper a novel IEEE 802.11e HCCA scheduler, Immediate Dynamic TXOP HCCA (IDTH) is presented. It integrates a bandwidth reclaiming scheme with a real-time HCCA scheduling algorithm in order to make unused resources available for variable bit rate traffic streams with more stringent QoS requirements. The analytical study and the simulations shown that is suitable to reduce the experienced delay and the packets queue lengths, especially in the case of highly variable traffic, and to improve the efficiency of resource management, without modifying the centralized scheduling policy nor the admission control.

\section{REFERENCES}

[1] P. Ansel, Q. Ni, and T. Turletti. FHCF: A simple and efficient scheduling scheme for IEEE 802.11e wireless LAN. Mobile Networks and Applications, 11(3):391-403, June 2006.

[2] T. P. Baker. Stack-based scheduling for real-time processes. Real-Time Syst., 3(1):67-99, 1991.

[3] G. Boggia, P. Camarda, L. G. Grieco, and S. Mascolo. Feedback-based control for providing real-time services with the IEEE 802.11e MAC. IEEE/ACM Trans. Net., 2(15):323-333, Apr. 2007.
[4] G. Cecchetti and A. L. Ruscelli. Real-Time support for HCCA function in IEEE 802.11e networks: a performance evaluation. Security and Communication Networks, 2010. Special Issue on Security for QoS Assured Wireless Networks.

[5] G. Cecchetti, A. L. Ruscelli, and F. Checconi. W-CBS: a scheduling algorithm for supporting QoS in IEEE 802.11e. In Proc. QSHINE, pages 1-7, Vancouver, British Columbia, Canada, 2007. ACM.

[6] C. Cicconetti, L. Lenzini, E. Mingozzi, and G. Stea. Design and performance analysis of the Real-Time HCCA scheduler for IEEE 802.11e WLANs. Computer Networks, 51(9):2311-2325, 2007.

[7] R. L. Cigno, L. Palopoli, and A. Colombo. Analysis of different scheduling strategies in $802.11 \mathrm{e}$ networks with multi-class traffic. In Proc. IEEE LCN, Dublin, Ireland, Oct. 2007.

[8] J. Cowling and S. Selvakennedy. A detailed investigation of the IEEE 802.11e HCF reference scheduler for VBR traffic. In Proc.13th ICC, pages 453-459, Chicago, US, Oct. 2004.

[9] Y. Fan and C. Huang. Real-Time traffic sheduling algorithm in WLAN. In Proc. 4GMF, San Diego, CA, 2005.

[10] H. Fattah and C. Leung. An overview of scheduling algorithms in wireless multimedia networks. IEEE Wireless, 9(5):76-83, Oct. 2002.

[11] A. Grilo, M. Macedo, and M. Nunes. A service discipline for support of IP QoS in IEEE 802.11 networks. In Proc. PWC, Laapenranta, Finland, Aug. 2001.

[12] A. Grilo, M. Macedo, and M. Nunes. A scheduling algorithm for QoS support in IEEE 802.11e networks. IEEE Wireless Comm., 10(3):36-43, June 2003.

[13] A. Grilo and M. Nunes. Performance evaluation of IEEE 802.11e. In 13th IEEE PIMRC, volume 1, pages 511-517, Lisboa, Portugal, Sept. 2002.

[14] http://traces.eas.asu.edu/, 2005.

[15] IEEE802.11. Wireless LAN medium access control (MAC) and physical layer (PHY) specification, 2007

[16] IEEE802.11e. 802.11e-2005 IEEE standard for information technology telecommunications and information exchange between systems local and metropolitan area networks specific requirements part 11: Wireless LAN medium access control (MAC) and physical layer (PHY) specifications: Amendment 8: Medium access control (MAC) quality of service enhancements, 2005.

[17] I. Inanc, F. Keceli, and E. Ayanoglu. An adaptive multimedia QoS scheduler for IEEE 802.11e wireless LANs. In Proc. IEEE ICC, Istanbul, Turkey, June 2006.

[18] C. Kuan and K. Dimyati. Utilization model for HCCA/EDCA mixed mode in IEEE 802.11e. ETRI Journal, 29(6):829-831, Dec. 2007.

[19] W. K. Lai, C. Shien, and C. Jiang. Adaptation of HCCA/EDCA ratio in IEEE 802.11 for improved system performance. Int. J. on Innovative Computing, Inf. and Contr., 5(11(B)):4177-4188, Nov. 2009.

[20] P. Larcheri and R. L. Cigno. Scheduling in 802.11e: Open-loop or closed-loop? In Proc. IFIP WONS, Les Mnuires, France, Jan. 2006.

[21] C. L. Liu and J. W. Layland. Scheduling algorithms for multiprogramming in a hard-real-time environment. J. ACM, 20:46-61, Jan. 1973.

[22] S. Lu, V. Bharghavan, and R. Srikant. Fair scheduling in wireless packet networks. IEEE/ACM Trans. Net., 7(4):473-489, Aug. 1999.

[23] S. Mangold, S. Choi, P. May, O. Klein, G. Hiertz, and L. Stibor. IEEE 802.11e wireless LAN for quality of service. In Proc. European Wireless, volume 1, pages 32-39, Florence, Italy, Feb. 2002. invited paper.

[24] L. Palopoli, R. L. Cigno, and A. Colombo. Control and optimization of HCCA 802.11e access scheduling. In Proc. IEEE CDC, New Orleans, LA, Dec. 2007.

[25] A. L. Ruscelli, G. Cecchetti, A. Alifano, and G. Lipari. Enhancement of QoS support of HCCA schedulers using EDCA function in IEEE 802.11e networks. Ad Hoc Networks, 10(2):147-161, 2012. Special Issue on Recent advances in analysis and deployment of IEEE 802.11e and IEEE 802.11p protocol families.

[26] V. A. Siris and C. Courcoubetis. Resource control for the EDCA and HCCA mechanisms in IEEE 802.11e networks. In Proc. WiOpt, pages 22-27, Boston, Massachusetts, 2006.

[27] D. Skyrianoglou, N. Passas, and A. K. Salkintzis. ARROW: An efficient traffic scheduling algorithm for IEEE 802.11e HCCA. IEEE Transaction on Wireless Communications, 5(12):3558-3567, Dec. 2006.

[28] S.-L. Tsao. Extending Earliest Due Date scheduling algorithms for wireless networks with location-dependent errors. In Proc. 52nd IEEE VTC-Fall, volume 1, pages 223-228, Boston, MA, Sept. 2000. 